\title{
Comparison of the Effect of Aerobic Exercise and Antidepressant Medications on Depression and Sexual Desire of Depressed Middle-Aged Women
}

\author{
Laleh Pourmousavi Khoshnab $^{1^{*}}$, Asghar Nikseresht ${ }^{1}$
}

\begin{abstract}
Objectives: The aim of this study was to evaluate the effect of 8 weeks of aerobic exercise on depression and sexual desire in middleaged women diagnosed with depression.

Materials and Methods: The present study is a quasi-experimental study. Sixty females volunteered to participate in this study and were randomly divided into 4 groups; group A was prescribed with antidepressant drug $(n=15)$, group B employed aerobic exercises $(n=15)$, group C combination of drug and aerobic exercise $(n=15)$ and group $D$ which was the control group $(n=15)$, respectively. Beck Depression Inventory was used in these 4 groups to assess their level of depression and sexual functioning. For statistical analysis of the data the $t$ test, one-way analysis of variance (ANOVA) and Bonferroni post hoc test was used.

Results: Comparison within groups showed a significant decrease in the pre-test on depression variables of the 3 groups; the drug group $(P=0.006)$, exercise $(P=0.011)$ and combination $(P=0.001)$ and also, regarding the variable libido, significant increase in the pre-test and post-test was observed. Regarding the sexual desire variable, results showed that only the aerobic exercise group had a significant increase in the pre-test and the post-test $(P=0.005)$. Comparison of the group variables showed a significant difference between the 4 study groups in both variables of depression and sexual desire.

Conclusion: Considering the effectiveness of exercise on depression, it is expected that aerobic exercise would be considered as an effective non-pharmacologic treatment with less side effect in reducing depression and increasing sexual desire.

Keywords: Aerobic exercise, Depression, Sexual dysfunction, Women
\end{abstract}

\section{Introduction}

Depression is the most common psychiatric complication of modern living and considering its high prevalence and health costs, has drawn the attention of mental health professionals. Women are more prone to this disorder in comparison to their male counterparts $(1,2)$. Depression is a chronic disorder that requires treatment with antidepressant drugs; but anti-depressant medications impose some unwanted side effects. Epidemiological evidence indicates that sexual dysfunction and dissatisfaction are the most common and disturbing side effects of anti-depressants (3-7). On the other hand, sexual dysfunction is a common complication of depression.

The normal human sexual response cycle is conventionally divided into 4 phases; desire, excitement, orgasm, and resolution. Any dysfunction in one or more of these phases may interfere with sexual function and lead to sexual disorders. This cycle is controlled by the interaction between the hypothalamic-pituitary-adrenal, autonomic nervous system, sex hormones (testosterone, estrogen and progesterone), neurotransmitters (serotonin, dopamine, noradrenalin and acetylcholine) and vasoactive peptides $(8,9)$.

Medical literatures suggest that regular exercise is important in maintaining women's health. Existing evidence indicated that physical exercise potentially boost and create positive changes in an individual's moods $(10,11)$. Studies have shown that participation in regular physical activity has been linked to higher levels of sexual satisfaction (4). However, only few studies have been conducted to evaluate the relationship between physical activity and sexual function. In women, physical activities decrease with age and women are less active than men and tend to spend their leisure time in less strenuous activities. In addition, evidence showed that physical exercise alters menopausal symptoms, sleep cycle, moods, stress, depression and musculoskeletal disorders in women (12-14).

Despite the high prevalence of sexual dysfunction in women, they choose not to divulge their problem for some cultural reasons and abstain from seeking medical solutions to their sexual problems $(3,15)$. Since depression is the most common mental disorder in women, therefore, this study aims to evaluate the effect of aerobic exercise and antidepressant medications on depression and sexual desire in middle aged women.

\section{Materials and Methods}

The present study is a quasi-experimental study. Our study population included 60 middle-aged women within the age range of 40-60 years diagnosed with depression 
in the city of Shiraz, Iran. They were randomly divided into 4 groups; group who was on sertraline $50 \mathrm{mg} / \mathrm{d}$ by physician prescribed as antidepressant treatment $(n=15)$, aerobic exercise group $(n=15)$, combination of aerobic exercise and antidepressant treatment $(n=15)$ and a control group $(n=15)$. After selecting the subjects, initially, the research topic, objectives, methods of implementation and its applicability were explained to the participants and then signed consents were taken. Participants' weight, height and body mass index were taken and recorded in the pre-test stage and results were presented in Table 1.

Then the participants in each group were instructed to fill up the Beck questionnaire which was intended to evaluate the level of depression and female sexual function index to evaluate sexual function. Participants in the aerobic group and the combination of drug and aerobic exercise group performed their exercises 3 sessions per week for 8 weeks, each session lasting for 40-60 minutes with a maximum heart rate intensity of $50 \%-70 \%$ on a treadmill. After the 8-week period, the participants in each group were again asked to fill up the same questionnaires.

\section{Statistical Methods}

Statistical analysis was done with the use of SPSS version 18. In order to determine the normal distribution of data, Kolmogorov-Smirnov test was used. Then paired $t$ test, analysis of variance (ANOVA) and Bonferroni post hoc test were used. $P \geq 0.05$ was considered as significance level.

\section{Results}

Our findings indicated a significant decrease in depression variable in the post-test in comparison to the pre-test in interventional groups by medication or exercise or both of them. Table 2 shows comparison of group variables before and after interventions; treatment by sertraline group $(P=0.006)$, aerobic exercise $(P=0.011)$, and combination group $(P=0.001)$. For sexual desire comparison, our results showed a significant increase only in the aerobic exercise group in the post-test in comparison to the pre- test. Table 3 shows the results of variables being compared during the 8 weeks of study. Significant difference was shown between the 4 groups on 2 variables of depression $(P=0.001)$ and sexual desire $(P=0.003)$, respectively.

Table 4 shows the results of Tukey test. Results of the study indicated that the level of depression in the medication and the combination groups $(P=0.006)$, medication and control groups $(P=0.036)$, aerobic and combination groups $(P=0.006)$, aerobic and control groups $(P=0.036)$ and combination and control groups $(P=0.0001)$ had significant statistical difference.

\section{Discussion}

The present study was designed to examine the effect of aerobic exercise and sertraline as antidepressant drug on depression and sexual desire of depressed middle-aged women.

Depression was significantly reduced in the post-test in comparison to the pre-test by medication and exercise or combination of both. Results of this study with regards to the use of anti-depressant drug alone in depressed women was consistent with the results of the studies conducted by El Shamy et al (16), but the results of our study were inconsistent with the results of the studies conducted by Zarshenas et al (17) and Hekmati et al (18).

In case of depressed women that only performed aerobic exercises, our results were consistent with the results of the studies conducted by Martinson et al (19) and Zarshenas et al (17), showing that performance of aerobic exercise alone does not exert positive effect on the depression of these depressed women.

Results of some researches indicated that long-term aerobic exercise can increase the plasma concentration of free tryptophan causing an increase in its synthesis and release in the blood circulation (14). There is a possibility that depressed women who performed aerobic exercises throughout this study, had an increase in the concentration of free tryptophan in their plasma and this could play a decisive role in improving depression in these subjects. In the case of simultaneous use of antidepressant drugs

Table 1. Participants Profile

\begin{tabular}{|c|c|c|c|c|}
\hline \multirow{2}{*}{ Group } & \multicolumn{4}{|c|}{ Variables } \\
\hline & Age (y) & Height $(\mathrm{cm})$ & Weight (kg) & BMI $\left(\mathrm{kg} / \mathrm{m}^{2}\right)$ \\
\hline Drug therapy & $50.6 \pm 5.86$ & $164 \pm 6.35$ & $64.31 \pm 6.88$ & $23.85 \pm 2.68$ \\
\hline Aerobic exercise & $50.27 \pm 7.19$ & $164.73 \pm 5.52$ & $64.58 \pm 6.6$ & $23.46 \pm 2.13$ \\
\hline Combination (drug and aerobic exercise) & $52 \pm 6.26$ & $166.6 \pm 6.73$ & $64.83 \pm 7.44$ & $24.12 \pm 3.87$ \\
\hline Control & $51.87 \pm 6.92$ & $165.07 \pm 7.07$ & $67.2 \pm 7.38$ & $24.49 \pm 1.75$ \\
\hline
\end{tabular}

Table 2. Comparison of Group Variables Before and After Intervention

\begin{tabular}{|c|c|c|c|c|c|c|c|c|c|}
\hline Variables & Stage & Treatment Group & $P$ & Aerobic Exercise & $P$ & Combination & $P$ & Control & $P$ \\
\hline \multirow{2}{*}{ Depression } & Pre-test & $59.8 \pm 5.72$ & \multirow{2}{*}{0.006} & $28.87 \pm 6.06$ & \multirow{2}{*}{0.011} & $29.33 \pm 5.39$ & \multirow{2}{*}{0.001} & $29.2 \pm 4.87$ & \multirow{2}{*}{0.068} \\
\hline & Post-test & $28.2 \pm 6.21$ & & $27.27 \pm 6.51$ & & $24.8 \pm 6.3$ & & $30 \pm 4.37$ & \\
\hline \multirow{2}{*}{ Sexual desire } & Pre-test & $6.4 \pm 5.22$ & \multirow{2}{*}{0.219} & $6.13 \pm 8.27$ & \multirow{2}{*}{0.005} & $6.6 \pm 2.55$ & \multirow{2}{*}{0.074} & $5.93 \pm 2.28$ & \multirow{2}{*}{0.790} \\
\hline & Post-test & $5.8 \pm 1.93$ & & $8.27 \pm 1.53$ & & $6.6 \pm 2.55$ & & $5.87 \pm 2.77$ & \\
\hline
\end{tabular}


Table 3. Compares the Changes Between Variables During the 8 Weeks

\begin{tabular}{lccccc}
\hline Variables & Medication Group & Aerobic Exercise & Combination & Control & $P$ \\
\hline Depression & $1.92 \pm 1.6$ & $2.13 \pm 1.6$ & $4.53 \pm 3.24$ & $1.56 \pm 0.8$ & 0.001 \\
Sexual desire & $1.8 \pm 0.6$ & $2.47 \pm 2.13$ & $1.87 \pm 0.93$ & $1.83 \pm 0.07$ & 0.003 \\
\hline
\end{tabular}

Table 4. Results of Tukey Post Hoc Test

\begin{tabular}{lll}
\hline Study groups & Depression & Sexual Desire \\
\hline Treatment group & & \\
$\quad$ Aerobic exercise & 1 & 0.003 \\
Combination & 0.006 & 0.25 \\
$\quad$ Control & 0.036 & 1 \\
Aerobic exercise group & & \\
$\quad$ Combination & 0.006 & 0.65 \\
$\quad$ Control & 0.036 & 0.025 \\
Combination group & & \\
Control & 0.0001 & 1 \\
\hline
\end{tabular}

and aerobic exercises it can be argued that the positive effect of aerobic exercise on some physiological indices of the hypothalamic-pituitary-adrenal axis may relate to the improvement of depression. Increased secretion of beta-endorphins, monoamines in the brain, improvement in the oxygen supply to the brain and significant decrease in stressors are the physiological changes brought about by the positive effect of exercise on the hypothalamic-pituitary-adrenal axis functioning and secretion of cortisol, resulting in improvement of depression indicators $(8,20)$.

Considering the increased levels of neurotransmitters serotonin, norepinephrine and dopamine as an effect of anti-depressant drug and also, performing aerobic exercises is associated with more favorable results in comparison to the other groups in reducing the scores of depression variables in the post-test in comparison to pre-test scores.

Comparison of depression variables between the 4 groups indicated a statistically significant difference. Results of our study and the study conducted by George et al. (20) and Chu et al (21) were consistent. Comparison between the 4 groups indicated that the combination group had gained better results in comparison to other groups in improving depression and this is probably due to the fact that this group benefitted more on the simultaneous effect of drug and exercise.

Regarding the variable of sexual desire, a significant increase was only observed in the exercise group in the post-test in comparison to the pre-test. Our results were consistent with the research conducted by Mojdeh and Zeighami Mohamadi (22). Depression is associated with sexual dysfunction in both genders $(9,23)$. As expected, use of antidepressant drugs has been shown to decrease the sexual desire of depressed patients.

Our study results in regard to the effect of aerobic exercises on sexual desire were consistent to the study conducted by Lorenz and Meston (14). Increased blood flow and improved self-confidence are factors affected positively by exercise in sexual desire $(5,24)$. With regards to the simultaneous use of antidepressants and aerobic exercise, the findings in our study were consistent to the findings of the studies conducted by Lorenz and Meston (14) and Cabral et al (12).

Despite the higher prevalence of sexual dysfunction in women, majority of these women prefer not to seek medical solutions to their problem due to cultural reasons. Inattention to this problem would have a negative effect on their physical, mental and social health $(3,15)$; so regular exercise could be a good advice for their general health and improvement in sexual indices.

In comparing the sexual desire variable, our results indicated that significant statistical differences existed between medication group and aerobic exercise group and also between aerobic exercise group and the control group. Depression can interfere with the ability to enjoy sexual activity. Depression imposes very expensive treatments and on the other hand it would act as catalyst to sexual problems. Loss of libido, changes in reaching orgasm, decreased frequency of sexual intercourse and sexual dissatisfaction are common complications. A depressed person feels persistent numbness, frustration, helplessness, worthlessness and guilt. Generally they detach themselves from their work and other activities that they previously enjoyed including the loss of sexual activity $(4,25)$. As what has been observed in group comparison, results indicated that aerobic exercise group showed better results in comparison to the other groups in the improvement of sexual desire and this probably may be due to the fact that this group did not use any anti-depressant drugs.

As can be observed, the use of anti-depressant drugs decreases sexual desire and on the contrary, aerobic exercise can increase sexual desire but this increase was not significant and it seems to be due to the effect of anti-depressant drugs. Middle aged women are usually prescribed anti-depressants for a long period of time and prolonged use of these drugs would result in an adverse effect on their sexually. In order to counter this adverse effect, it is important to educate these individuals to perform regular exercise when taking anti-depressant drugs in order to maintain their minimal sexuality and emotional stability necessary to achieve a normal life. However, more study and more time to examine the effect of aerobic exercise on depressed women's sexual desire is required.

\section{Conclusion}

Based on the results of the present study, we can conclude that anti-depressant drugs and aerobic exercise alone and simultaneous use of anti-depressant drugs and exercise can improve depression; however, simultaneous use of anti-depressant drugs and aerobic exercises play a more ac- 
tive role in improving depression. As to the variable, sexual desire, the group of aerobic exercise without anti-depressant drugs gained better results. Considering the effectiveness of aerobic exercise on depression, it is expected that this type of training along with anti-depressant drugs be strictly recommended to improve depression.

\section{Ethical Issues}

Ethical considerations including the acquisition of informed consent from all participants prior to the study, maintaining the anonymity of the participants, confidentiality of information and the right to withdraw from the study were considered. Also, the study were adapted according to the ethic codes of Azad University.

\section{Conflict of Interests}

The authors declare no conflict of interests.

\section{Financial Support}

The researchers received no financial support or grant from any funding agency in the public and commercial sectors.

\section{Acknowledgments}

None to be declared.

\section{References}

1. Shokri F, Khoshnam E, Nikseresht A. The effect of eight weeks of aerobic exercise on depression, anxiety and sleep disorders in middle-aged women. Eur J Exp Biol. 2014;4(1):557-559. doi:10.5812/ircmj.16408.

2. Yeh HS, Lin WL, Chuang K, et al. Effects of music aerobic exercise on depression and brain-derived neurotrophic factor levels in community dwelling women. BioMed Res Int. 2015;2015:135893. doi:10.1155/2015/135893

3. Baldwin SD. Depression and sexual dysfunction. Br Med Bull. 2001;57(1):81-99.

4. Franchini L, Ciraci M, Redaelli C, Colombo C. Sexual dysfunction during long-term treatment with antidepressants in unipolar disorder: clinical and management aspects. J Psycopathol. 2012;18:131-137.

5. Lin CF, Juang YY, Wen JK, Liu CY, Hung CI. Correlations between sexual dysfunction, depression, anxiety, and somatic symptoms among patients with major depressive disorder. Chang Gung Med J. 2012;35(4):323-331.

6. Duman S. Effect of step aerobic exercise on body composition and depression levels of women in menopause. Middle-East J Sci Res. 2013;16(2):172-178.

7. Harsodaa MJ. Comparative study of change in physical activity on exercise performance and mental wellbeing. Int J Appl Exercise Physiol. 2014;3(1):63-43.

8. Outhoff K. Antidepressant-induced sexual dysfunction. S Afr Fam Pract. 2009;51(4):298-302. doi:10.1080/20786204 .2009 .10873868 .
9. Higgins A, Nash M, Lynch MA. Antidepressant-associated sexual dysfunction: impact, effects, and treatment. Drug Healthc Patient Saf. 2010;2:141-150. doi:10.2147/DHPS. S7634.

10. Meston CM. Sympathetic nervous system activity and female sexual arousal. Am J Cardiol. 2000;86(2):30-34.

11. Strohle A. Physical activity, exercise, depression and anxiety disorders. J Neural Transm. 2009;116(6):777-784.

12. Cabral PU, Canário AC, Spyrides MH, et al. Physical activity and sexual function in middle-aged women. Revista da Associação Médica Brasileira 2014;60(1):47-52.

13. Daniels KA, Niekerk LR. The influence of a moderate aerobics programme on the body self-image of women in middle adulthood. S Afr J Sports Med. 2011;23(4):106-110.

14. Lorenz TA, Meston CM. Acute exercise improves physical sexual arousal in women taking antidepressants. Ann Behav Med. 2012;43(3):352-61. doi:10.1007/s12160-011-9338-1.

15. Hamilton LD, Rellini AH, Meston CM. Cortisol, sexual arousal, and affect in response to sexual stimuli. J Sex Med. 2008;5(9):2111-2118.

16. El-Shamy FF, El-Kosery E, El-Bigawy A. Effect of aerobic exercise on postmenopausal women with major depression. Br J Appl Sci Technol. 2013;3(2):214-219.

17. Zarshenas S, Houshvar P, Tahmasebi A. The effect of shortterm aerobic exercise on depression and body image in Iranian women. Depress Res Treat. 2013;2013:132684. doi:10.1155/2013/132684.

18. Hekmati P, Hojate H, Sharefneya H, Akhonzadi G. The effect of regular exercise on depression in the elderly. Journal of Health Education and Health Promotion 2013;3:32-23.

19. Martinson EW, Hoffart A, Solberg F. Comparing aerobic with non aerobic forms of exercise in the treatment of clinical depression: a randomized trial. Compr Psychiatry. 1989;30(4):324-331.

20. George J, Chandran M, Sandesh TS. A comparative study to evaluate the effectiveness of resisted exercises verses antidepressant medication in young adult males diagnosed with major depressive disorder. J Biol Agric Healthc 2012;2:27-34.

21. Chu IH, Buckworth J, Kirby TE, Emery CF. Effect of exercise intensity on depressive symptoms in women. Ment Health Phys Act. 2009;2(1):37-43.

22. Mojdeh F, Zeighami Mohamadi S. The relationship between depression and sexual function index in married women (Persian). Sci J Hamedan Nursing Midwifery 2013;21(1):41-51.

23. Jespersen S. Antidepressant induced sexual dysfunction Part1: epidemiology and clinical presentation. Afr J Psychiatry. 2006;9(1):24-27.

24. Swan JA, Hyland PH. A review of the beneficial mental health effects of exercise and recommendations for future research. Psychology Soc. 2012;5(1):1-15.

25. Niteen A, Neena S. Effect on sexual dysfunction in depressed females after treatment with SSRI antidepressants. Int J Recent Trends Sci Technology. 2014;12:24-27.

Copyright (c) 2017 The Author (s); This is an open-access article distributed under the terms of the Creative Commons Attribution License (http://creativecommons.org/licenses/by/4.0), which permits unrestricted use, distribution, and reproduction in any medium, provided the original work is properly cited. 